\title{
PRODUÇÃO DE HIDROGÊNIO VIA REFORMA A VAPOR DO ETANOL: ANÁLISE TERMODINÂMICA
}

\author{
G. CAMBAÚVA ${ }^{1}$, S. C. M. MIZUNO ${ }^{1}$, J. B. O. SANTOS ${ }^{1}$ \\ ${ }^{1}$ Universidade Federal de São Carlos, Departamento de Engenharia Química \\ E-mail para contato: joaobatista@ufscar.br
}

\begin{abstract}
RESUMO - O processo de reforma a vapor do etanol mostra-se como uma excelente rota de produção de hidrogênio, pois utiliza matéria-prima renovável e de baixo custo. $\mathrm{O}$ consumo de hidrogênio tem aumentado nos últimos anos, sendo utilizado principalmente no refino do petróleo, produção de amônia e em células a combustível. A produção de hidrogênio via reforma a vapor do etanol foi avaliada com uma análise termodinâmica do processo utilizando o software Aspen Plus. As simulações foram conduzidas variando-se as condições de pressão e temperatura do processo, além da vazão de alimentação da água e etanol na corrente de alimentação do reator. Foi possível observar que elevada razão água/etanol aumenta a produção de hidrogênio. $\mathrm{O}$ aumento da temperatura também aumenta a formação de hidrogênio e o aumento da pressão não é favorável ao processo.
\end{abstract}

\section{INTRODUÇÃO}

A matriz energética mundial fundamenta-se na utilização de combustíveis fósseis, os quais são responsáveis pela liberação de gases associados ao efeito estufa e ao aquecimento global. Neste panorama, observa-se uma contínua redução das reservas petrolíferas e um aumento das políticas de controle ambiental, e assim, as principais discussões do século XXI giram em torno de se encontrar alternativas às fontes energéticas não renováveis.

Entre as principais alternativas energéticas pode-se destacar o hidrogênio. Além da combustão de hidrogênio gerar somente vapor d'água, trata-se de uma energia isenta de impurezas, com uma elevada taxa de eficiência energética por unidade de massa se comparada com outros combustíveis comumente empregados. Atualmente, a principal aplicação do hidrogênio em escala industrial é no refino do petróleo, em reações de hidrodesoxigenação, hidrodessulfurização e hidrodesnitrogenação (Elizalde e Anchyeta, 2009). A principal rota produtiva do hidrogênio utilizado em refinarias de petróleo é a reforma a vapor do gás natural (Haryanto et al., 2005), que é realizada em altas pressões e temperaturas. O elevado consumo mundial de energia deve promover um aumento da produção de hidrogênio nos próximos anos. Além de sua aplicação no refino do petróleo, o hidrogênio também é utilizado em células a combustível e poderá ser empregado como combustível de automóveis.

Alternativamente à reforma a vapor do gás natural, tem-se a reforma a vapor do etanol como via de produção de hidrogênio, que utiliza uma matéria-prima renovável (etanol), de fácil armazenamento e transporte seguro. A reforma a vapor do etanol (RVE, reação 1) ocorre em condições menos drásticas (menores pressões e temperaturas) do que a reforma a vapor do 
metano. Além disso, o Brasil possui uma das melhores tecnologias do mundo na produção de etanol, contando com uma boa infraestrutura de fabricação, armazenamento e distribuição.

$$
\mathrm{C}_{2} \mathrm{H}_{5} \mathrm{OH}_{(\mathrm{g})}+3 \mathrm{H}_{2} \mathrm{O}_{(\mathrm{g})} \rightleftarrows 2 \mathrm{CO}_{2(\mathrm{~g})}+6 \mathrm{H}_{2(\mathrm{~g})}
$$

As condições operacionais do processo de produção de hidrogênio via RVE afetam fortemente a composição de saída dos produtos. A literatura reporta que a conversão de etanol e subprodutos de carbono aumenta com o aumento da temperatura e razão de alimentação água/etanol. Portanto, a otimização das variáveis de processo como pressão, temperatura e razão de alimentação água/etanol se configura como a principal rota de aumento da produtividade de hidrogênio no processo (Compagnoni et al., 2016).

Estudos termodinâmicos da RVE mostraram que a reação acontece em temperaturas acima de $500 \mathrm{~K}$, com formação de metano, água, CO, CO2 e hidrogênio (Vasudeva et. al., 1996; Laborde e Garcia, 1991). Acetaldeido e etileno também são formados, mas são considerados como compostos intermediários (Fishtik et al., 2000), pois são transformados rapidamente em outros produtos quando o tempo espacial é elevado.

O mecanismo da RVE tem sido estudado utilizando-se vários catalisadores (Zanchet et al., 2015; Li et. al., 2016) e os resultados mostram que o etanol é inicialmente convertido para acetaldeido, que em seguida é desidrogenado na superfície do catalisador formando espécies $\mathrm{H}_{\mathrm{x}} \mathrm{C}-\mathrm{CH}_{\mathrm{x}}$. A reação do etanol sobre o suporte pode levar a produção de etileno, que é um precursor da formação de carbono sobre o catalisador. A quebra da ligação C-C leva a formação de $\mathrm{CH}_{4}, \mathrm{CO}$ e $\mathrm{CO}_{2}$.

O maior problema encontrado na RVE é a formação de carbono na superfície do catalisador, que leva a desativação do catalisador. A estequiometria da reação sugere que três mols de água são consumidos para cada mol de etanol. Entretanto, razões molares de água/etanol acima de três tem sido empregadas com o objetivo de evitar a desativação do catalisador. Outro fator importante é o gasto com energia, visto que uma maior quantidade de água significa um custo maior com energia.

Para entender o processo de produção de hidrogênio via RVE, e desenvolver um catalisador eficiente para o processo, são necessários estudos detalhados da termodinâmica e cinética das etapas envolvidas na reação (1). Portanto, para entender o efeito das variáveis de processo sobre a produção de hidrogênio, uma análise termodinâmica da RVE foi realizada no software Aspen Plus.

\section{OBJETIVOS}

O objetivo deste trabalho é realizar uma análise termodinâmica da RVE e verificar a influência da razão de alimentação água/etanol (entre 3 e 5), temperatura (entre 500 e $700{ }^{\circ} \mathrm{C}$ ) e pressão (entre 1 e 10 atm), sobre a produção de hidrogênio. 


\section{METODOLOGIA}

A análise termodinâmica da RVE foi realizada no software ASPEN Plus. No ambiente de simulação do Aspen Plus utilizou-se o reator de Gibbs, que é capaz de simular um equilíbrio químico monofásico ou multifásico, em que é possível especificar temperatura, e pressão, incluindo variação de entalpia do processo. A análise termodinâmica foi realizada com o método da minimização da energia livre de Gibbs, que é capaz de estimar o equilíbrio químico, mediante as restrições dos balanços de massa dos compostos envolvidos, sem que seja necessário apresentar as reações químicas.

O fluxograma do processo utilizado no ASPEN Plus é apresentado na Figura 1. A alimentação consiste em etanol e água, enquanto a corrente de saída do reator de Gibbs consiste nos produtos formados na reação. Baseado na literatura, os principais produtos formados na reforma a vapor do etanol são: $\mathrm{H}_{2} \mathrm{O}$ (água), $\mathrm{CO}$ (monóxido de carbono), $\mathrm{CO}_{2}$ (dióxido de carbono), $\mathrm{H}_{2}$ (hidrogênio), $\mathrm{CH}_{4}$ (metano), $\mathrm{C}_{2} \mathrm{H}_{5} \mathrm{OH}$ (etanol), $\mathrm{CH}_{3} \mathrm{CHO}$ (acetaldeído), $\mathrm{C}_{2} \mathrm{H}_{4}$ (etileno) e $\mathrm{C}$ (carbono). As simulações consistiram na utilização de uma corrente de alimentação de água/etanol na razão molar de 3 até 5, pressões entre 1 e $10 \mathrm{~atm}$, e temperatura entre 500 e $700{ }^{\circ} \mathrm{C}$.

Figura 1 - Fluxograma do processo no reator de Gibbs.

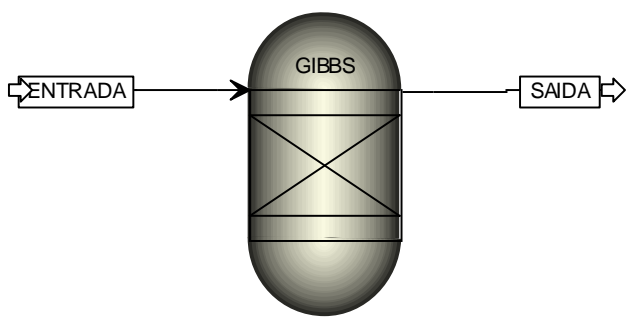

Utilizou-se a análise de sensibilidade do software para verificar a influência da razão água/etanol na entrada do reator, pressão do reator e temperatura sobre a formação dos produtos.

\section{RESULTADOS E DISCUSSÃO}

A Figura 2 mostra os resultados da simulação da RVE conduzida a $600^{\circ} \mathrm{C}$ e pressão de 1 atm, variando a razão molar de água/etanol entre 3 e 5 . O principal produto formado é o $\mathrm{H}_{2}$ e a sua quantidade aumenta com o aumento da razão água/etanol. Resultados experimentais sugerem que a utilização de uma elevada razão molar de água/etanol minimiza a formação de carbono sobre a superfície do catalisador e aumenta a quantidade de $\mathrm{H}_{2}$ (Compagnoni et al., 2016; Matteo et al., 2016). A formação de CO permanece praticamente constante, ou seja, não é afetada pela quantidade de água na alimentação, enquanto a formação de $\mathrm{CO}_{2}$ aumenta levemente com o aumento da quantidade de água e a produção de $\mathrm{CH}_{4}$ diminui (Figura 2). $\mathrm{O}$ aumento da quantidade de $\mathrm{CO}_{2}$ deve-se a reação de deslocamento gás-água $\left(\mathrm{CO}+\mathrm{H}_{2} \mathrm{O} \rightleftarrows \mathrm{CO}_{2}+\mathrm{H}_{2}\right)$. 
A influência da razão água/etanol na produção de $\mathrm{H}_{2}$ via reforma do etanol foi estudada por Campagnoni et al. (2016). Dados experimentais mostraram que um aumento na razão água/etanol promove um aumento na conversão de etanol e uma diminuição na razão $\mathrm{CO} / \mathrm{CO}_{2}$ produzido. Já a formação de $\mathrm{CH}_{4}$, se mostrou muito mais dependente das condições de operação (temperatura e velocidade espacial do gás), ou seja, com pouca sensibilidade à variação de razão água/etanol. Os resultados apresentados neste trabalho são similares ao obtidos por Campagnoni et al. (2016).

Figura 2 - Influência da razão de alimentação água/etanol na formação de produtos na RVE conduzida a $1 \mathrm{~atm}$ e $600{ }^{\circ} \mathrm{C}$.

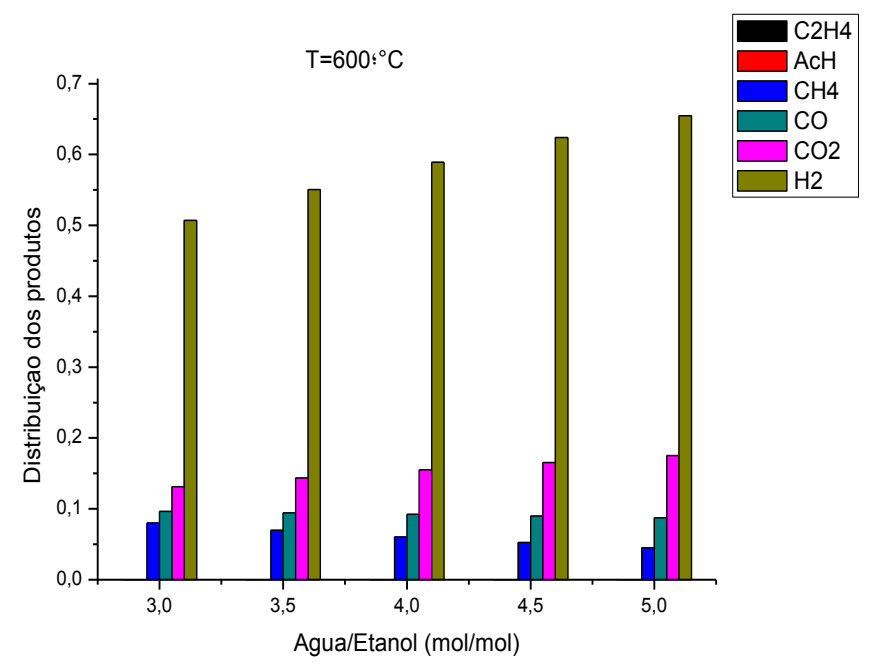

A Figura 3 exibe os resultados da simulação da RVE conduzida com uma razão de alimentação água/etanol igual a 4 e pressão de 1 atm, variando a temperatura entre 550 e $650^{\circ} \mathrm{C}$. O principal produto formado é o $\mathrm{H}_{2}$ e a sua quantidade aumenta com o aumento da

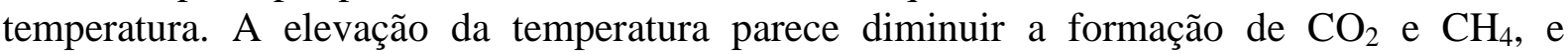
aumentar a quantidade de CO (Figura 3). Isso pode ser explicado pela ocorrência simultânea das reações de reforma a vapor do metano $\left(\mathrm{CH}_{4}+\mathrm{H}_{2} \mathrm{O} \rightleftarrows \mathrm{CO}+3 \mathrm{H}_{2}\right)$, deslocamento gás-água e reforma seca do metano do metano $\left(\mathrm{CH}_{4}+\mathrm{CO}_{2} \rightleftarrows 2 \mathrm{CO}+2 \mathrm{H}_{2}\right)$.

A influência da temperatura na produção de $\mathrm{H}_{2}$ via reforma do etanol também foi estudada por Campagnoni et al. (2016), que mostraram que um aumento na temperatura promove um aumento na conversão de etanol, aumento na razão $\mathrm{CO} / \mathrm{CO}_{2}$ produzido e diminuição da formação de $\mathrm{CH}_{4}$. Os resultados apresentados neste trabalho são semelhantes aos apresentados na literatura (Campagnoni et al., 2016).

Os resultados da simulação da reforma a vapor do etanol conduzida com uma razão de alimentação água/etanol=4, variando a pressão entre 1 e 9 atm e temperatura entre 100 e $600^{\circ} \mathrm{C}$ são apresentados na Figura 4. Pode-se observar que a produção de hidrogênio diminui com o aumento da pressão. De fato, resultados experimentais obtidos por Aupretre et al. (2005) indicam que pressões elevadas não favorecem a produção de hidrogênio. 
Figura 3 - Influência da temperatura na formação de produtos na RVE realizada a pressão atmosférica com razão água/etanol de 4.

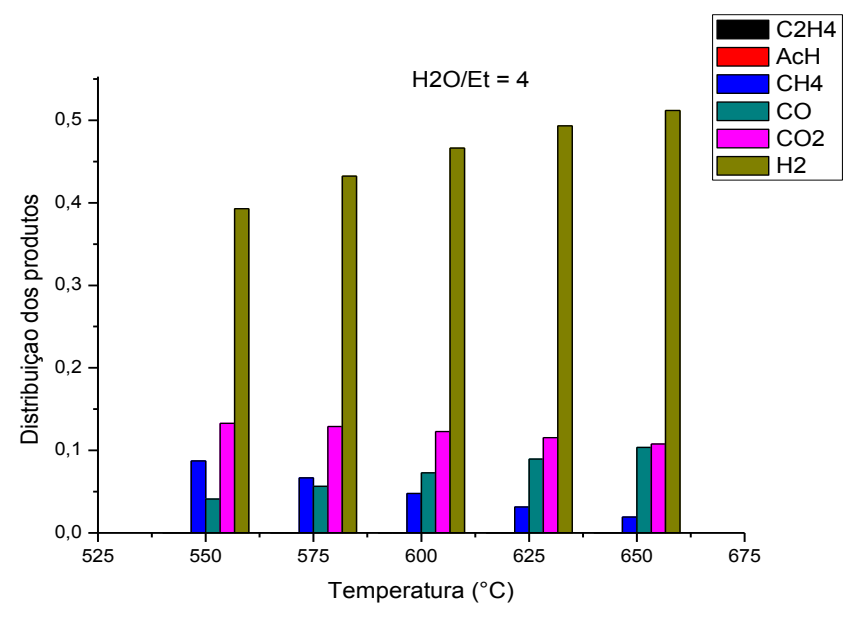

Figura 4 - Influência da pressão total na produção de hidrogênio na RVE.

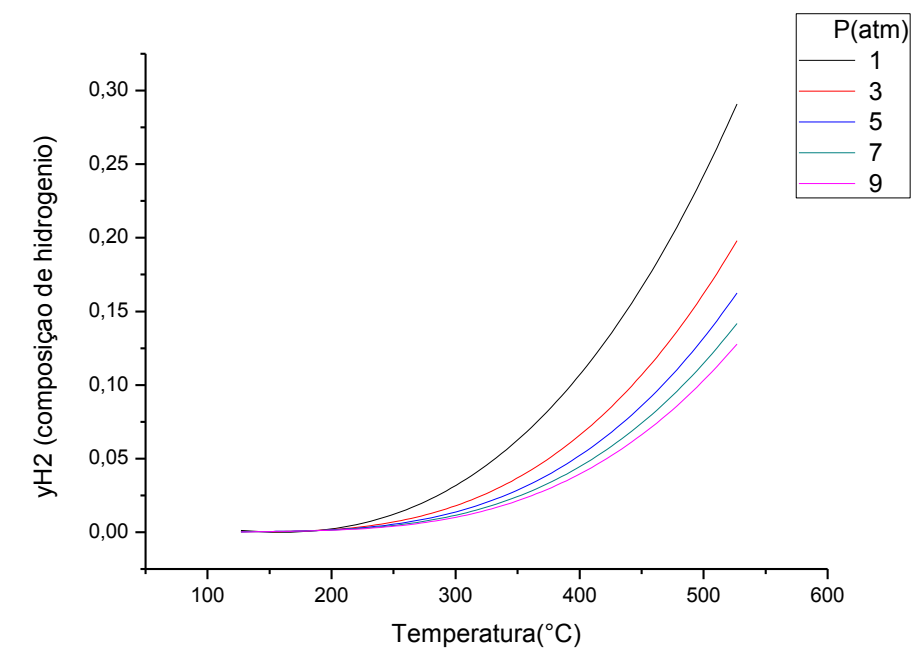

A influência da pressão na produção de $\mathrm{H}_{2}$ via reforma do etanol também foi estudada por García et al. (1991). Dados experimentais mostraram que uma diminuição na pressão promove um aumento da composição de hidrogênio produzido. Os resultados exibidos neste trabalho são semelhantes aos mostrados por García et al. (1991).

\section{CONCLUSÕES}

O modelo proposto no trabalho se mostrou muito próximo aos dados experimentais reportados na literatura. Além disso, forneceu importantes informações para a otimização deste processo. 
A análise termodinâmica mostrou que a produção de hidrogênio aumenta com o aumento da temperatura e da razão água/etanol, enquanto a pressão deve ser mantida baixa (1 atm) durante a reação.

\section{REFERÊNCIAS}

AUPRETRE, F.; DESCORME, C.; DUPREZ, D.; CASANAVE, D.; UZIO, D. , Ethanol steam reforming over $\mathrm{Mg}_{\mathrm{x}} \mathrm{Ni}_{1-\mathrm{x}} \mathrm{Al}_{2} \mathrm{O}_{3}$ spinel oxide-supported $\mathrm{Rh}$ catalysts, Journal of Catalysis, v. 233, p. 464-477, 2005.

COMPAGNONI, M.; TRIPODI, A.; ROSSETTI, I. Parametric study and kinetic testing for ethanol steam reforming. Applied Catalysis B: Environmental., p. 899-909, 2016.

ELIZALDE, I.; ANCHYETA, J. Modeling the Simultaneous Hydrodesulfurization and Hydrocracking of Heavy Residue Oil by using the Continuos Kinetic Lumping Approach. Energy \& Fuels., v.26, n.4, p.1999-2004, 2009.

FISHTIK, I.; ALEXANDER, A.; DATTA, R.; GEANA, D., A thermodynamic analysis of hydrogen production by steam reforming of ethanol via response reactions, Int. J. Hydrogen Energy, vol. 25, no. 1, p. 31-45, 2000.

GARCÍA, E. Y.; LABORDE, M. A. Hydrogen production by the steam reforming of ethanol: thermodynamic analysis, Int. J. Hydrogen Energy., v.16, n. 5, p. 307-312, 1991.

HARYANTO, A.; FERNANDO, S.; MURALI, N.; ADHIKARI, S., Current status of hydrogen production techniques by steam reforming of ethanol: A review. Energy \& Fuels., v.19, n.5, p.2098-2106, 2005.

Li, D.; Li, X.; Gong, J., Catalytic Reforming of Oxygenates: State of the Art and Future Prospects, Chem. Rev., v. 116, p. 11529-11653, 2016.

VASUDEVA, K.; MITRA, N.; UMASANKAR, P.; DHINGRA, S.C.,Steam reforming of ethanol for hydrogen production: thermodynamic analysis, Int. J. Hydrogen Energy, v. 21, no. 1, p. 13-18, 1996.

ZANCHET, D.; SANTOS, J. B. O.; DAMYANOVA, S.; GALlO, J. M. R.; C. BUENO, J. M. Toward Understanding Metal-Catalyzed Ethanol Reforming, ACS Catalysis, v. 5, p. 38413863, 2015. 